Supporting Information

\title{
Super-Resolution Optical Lithography with DNA
}

Shi Ho Kim, Yu Liu, Conner Hoelzel, Xin Zhang* and Tae-Hee Lee*

Department of Chemistry, The Pennsylvania State University, University Park 16802

*Corresponding authors (xuz31@psu.edu and tx118@psu.edu) 


\section{Materials and Methods}

\section{Microscope slide preparation}

Biotin-Polyethyleneglycol(PEG)-silane (MW 3400, Laysan Bio, Arab, AL) was used to coat a clean quartz microscope slide in the following steps. A set of new quartz slides was washed thoroughly in acetone, dichloromethane, and methanol through sonication for 15 minutes each, followed by rinsing with deionized (DI, $>18 \mathrm{M} \Omega$ resistance) water. Upon thorough drying with pure $\mathrm{N}_{2}$ gas (purity $99.99 \%$ ), propane torch was used to flame the cleaned slides to remove any residual organic adsorbents. The slides were immersed in a $500 \mathrm{~mL}$ sulfuric acid mixed with $1 / 2$ pack of Nochromix (Sigma, St Louis MO) for overnight, followed by a thorough rinse with DI water. The slides were then blow-dried with $99.99 \% \mathrm{~N}_{2}$ before undergoing the following functionalization steps. Biotin-PEG-silane was dissolved in anhydrous acetonitrile (spectrophotometric grade, low water, OmniSolv, product no. AX0151-1) to a concentration of $0.5 \mathrm{mg} / \mathrm{mL}$ with sonication. Cleaned quartz slides were immersed in the biotin-PEG-silane solution for 20 minutes with sonication, followed by additional sonication in DI water twice for 5 minutes each to remove any unreacted biotin-PEG-silane. The resulting slides were blowdried with $99.99 \% \mathrm{~N}_{2}$ and stored under vacuum before use.

\section{DNA inter-strand crosslinker synthesis}

A DNA inter-strand crosslinker Cyanovinylcarbazole N-hydroxysuccinimide ester (CNV-NHS-ester) was synthesized based on the protocols previously reported ${ }^{1-2}$. Briefly, Cyanovinylcarbazole (CNV) was first synthesized by palladium catalyzed crosscoupling. Alkylation of CNV yielded a precursor compound, which was later deprotected using standard ester hydrolysis conditions. The deprotected cyanovinylcarbazole containing carboxylic acid was activated by coupling into an N-hydroxysuccinimide ester (NHS ester).

\section{Synthesis of 3-cynovinylcarbazole NHS ester (CNV-NHS-ester)}

Compound 3 in figure 2A (276.3 mg, $1 \mathrm{mmol}, 1$ eq.) and N-Hydroxysuccinimide (172.6 $\mathrm{mg}, 1.5 \mathrm{mmol}, 1.5$ eq.) were dissolved in anhydrous DMF (10 mL). 1-ethyl-3-(3Dimethylaminopropyl) carbodiimide $\mathrm{HCl}(\mathrm{EDC} \cdot \mathrm{HCl}, 211 \mathrm{mg}, 1.1 \mathrm{mmol}, 1.1 \mathrm{eq}$.$) was$ added in one portion at $0{ }^{\circ} \mathrm{C}$. The reaction mixture was stirred at room temperature for 12 h. DMF was removed by vacuum and $50 \mathrm{~mL}$ dichloromethane was added to dissolve the reaction mixture. The organic layer was then quickly washed with water and brine. The resulted solid was washed with ether and dichloromethane to afford white solid. All reagents and anhydrous solvents of commercial grade were used as received unless otherwise noted. Reactions were monitored by thin layer chromatography (TLC) analysis using Silicycle ${ }^{\circledR}$ glass sheets pre-coated with silica gel ( $\left.60 \AA\right)$ with detection by UV absorption ( $254 \mathrm{~nm}$ or $365 \mathrm{~nm}$ ). Flash column chromatography was performed using Silica Flash ${ }^{\circledR}$ F60 silica gel in the indicated solvent mixture. ${ }^{1} \mathrm{H}$ NMR and ${ }^{13} \mathrm{C}$ NMR spectra (Fig. S1) were obtained on a Bruker NMR $(500 / 126 \mathrm{MHz})$ spectrometer in DMSO solvent. Liquid chromatography - mass spectrometry (LC-MS) analysis (Fig. S1) 
was completed using a Thermo Scientific ${ }^{\mathrm{TM}}$ Vanquish LC system equipped with an Accucore Vanquish C18+ column $(50 \times 2.1 \mathrm{~mm}, 1.5 \mu \mathrm{m}$ particle size $)$ and a $\mathrm{Q}$ Exactive $^{\mathrm{TM}}$ Hybrid Quadrupole-Orbitrap Mass Spectrometer.

\section{DNA hairpin preparation}

The hairpin structure is made of three different ss-DNA strands - anchor, pin, and bond strands which were purchased (Integrated DNA Technologies Inc., Coralville IA). The sequences of the three strands are given in Supporting Information (Fig. S2). The NHSester modified crosslinker was conjugated to the pin strand that is modified with an amine group. The crosslinker was dissolved in $100 \%$ DMSO at a concentration of $9.71 \mathrm{mg} / \mathrm{mL}$. A 200-fold excess of crosslinker to DNA is mixed in a sodium bicarbonate buffer $(\mathrm{pH}$ $8.3,50 \mathrm{mM}$ ) at a room temperature and gently agitated for four hours. The resulting solution was stored in $-20{ }^{\circ} \mathrm{C}$ for overnight ( 8 hours). Phenol-chloroform extraction was carried out by adding an equal volume of phenol:chloroform:isoamyl alcohol mix $(25: 24: 1)$ to the solution and taking the water layer for 6 times followed by ethanol precipitation with chilled ethanol at $-80{ }^{\circ} \mathrm{C}$. The ethanol precipitated solution was spun down at $14,000 \mathrm{~g}$ in a temperature controlled centrifuge at $4^{\circ} \mathrm{C}$ for 30 minutes. The supernatant was carefully removed with pipette and the sediment was dried for 10 minutes in SpeedVac (Thermofisher Scientific, Watham MA). The purified pin strand was dissolved in $20 \mathrm{uL}$ DI a Tris buffer (final concentration $76 \mathrm{uM}$ ). The anchor and bond strand was dissolved in water to be stored at a $100 \mathrm{uM}$ concentration. Equimolar amounts of the three strands were mixed to make a $1 \mathrm{uM}$ final solution of $60 \mathrm{uL}$ Tris buffer $(\mathrm{pH}$ $7 / 5,10 \mathrm{mM}$ Tris, and $100 \mathrm{mM} \mathrm{NaCl})$. The mixture was annealed in a thermal cycler (MJ Mini, Bio-Rad, Hercules CA) via $34 \times 2.5^{\circ} \mathrm{C}$ steps from $90{ }^{\circ} \mathrm{C}$ to $5^{\circ} \mathrm{C}$.

\section{Immobilizing DNA hairpin on the surface}

A sample chamber was constructed between a PEGylated slide and a cleaned coverslip as was previously published ${ }^{3}$. Briefly, a slide with $0.5 \mathrm{~mm}$ diameter holes for solution injection and flow is covered and bonded with a coverslip using a double-sided tape. Tween 20 (Sigma, St Louis MO) was injected to the sample chamber and incubated for 10 minutes to reduce any non-specific binding. A $50 \mathrm{uL}$ solution of Streptavidin (Thermofisher Scientific, Watham MA) at $1 \mathrm{mg} / \mathrm{mL}$ in $10 \mathrm{mM}$ Tris (pH 7.5) was injected and incubated for 10 minutes. Upon rinsing the chamber with $50 \mathrm{uL} 10 \mathrm{mM}$ Tris $(\mathrm{pH}$ 7.5), DNA hairpin at $1 \mathrm{nM}$ was injected and incubated for 8 minutes. Protocatechuate dioxygenase (PCD, Sigma, St Louis MO, $0.4 \mathrm{u} / \mathrm{mL}$ ) and Protocatechuic acid (PCA, Sigma, St Louis MO, $4 \mathrm{mM}$ ) were injected to avoid premature photobleaching of the fluorophores during the patterning process.

\section{Polystyrene nanoparticle conjugation to DNA}

A $100 \mu \mathrm{L}$ solution of neutravidin coated polystyrene nanoparticles (FluoSpheres, $0.5 \%$ solids, F8780, Thermofisher Scientific, Watham MA) was mixed and gently agitated with a biotin labeled DNA strand at a 1:2 ratio at a room temperature. The unreacted DNA was filtered out with a spin column with a $32 \mathrm{k}$ Mw cut. Briefly, a $4 \mathrm{~mL} 10 \mathrm{mM}$ Tris (pH 7.5) buffer containing $10 \mathrm{mM} \mathrm{NaCl}$ was added to the mixture and spin down until $100 \mathrm{uL}$ 
of the mixture is left. This filtering was repeated for 5 times. The same buffer was added to make the final volume of $400 \mathrm{uL}$ and the resulting nanoparticles were stored in $4^{\circ} \mathrm{C}$. A 5uL DNA-coated nanoparticle solution diluted to $40 \mathrm{uL}$ with the identical buffer was used each time to incubate on a patterned surface to create a particle assembly.

\section{Center determination of a one-dimensional (1D) or two-dimensional (2D) fluorescence dot image}

The center of a line pattern of fluorescence was determined by fitting the crosscorrelation between the line pattern and its reverse to a $4^{\text {th }}$ order polynomial. The separation between the point where the polynomial has the maximum value and the halfway of the line was determined first. One-half of this value is the deviation of the exact center of this 1D pattern from the halfway of the line. To determine the center of a 2D fluorescence dot image, the above process was carried out twice for $x$ - and $y$-axes. First, 5 pixel-lines were integrated along the $y$-axis to form a 1D $x$-array of the pattern. This 1D array was fitted as is described above to determine the $x$ center of the pattern. This process was repeated along the $y$-axis to determine the $y$ center of the pattern.

\section{SOLiD resolution limit}

The resolution of SOLiD is limited by (i) the photobleaching lifetime of the FRET donor, (ii) the surface density of DNA hairpins, and (iii) the wavelength of the FRET excitation beam. The super-resolution limit of FRET acceptor does not limit the SOLiD resolution because we can elongate the camera exposure time to collect enough photons to make this limit exceed the other limits listed above. Assuming that the limit set by (ii) can be controlled to exceed that by (i), the resolution limit $\mathrm{R}$ is approximately

$$
R \approx \frac{1}{2} \sqrt{\frac{\tau_{\text {folded }} S_{\text {FRET }}}{\tau_{\text {photobleaching }}}}=\frac{1}{2} \sqrt{\frac{\pi E}{2 \tau_{\text {photobleaching }}}} \frac{\lambda_{\text {FRET }}}{N . A .}
$$

, where $\tau_{\text {folded }}$ is the folded state lifetime of a hairpin, $S_{F R E T}$ is the beam area of FRET excitation, $\tau_{\text {photobleaching }}$ is the photobleaching lifetime, $E$ is the camera exposure time, $\lambda_{F R E T}$ is the FRET excitation wavelength, and N.A. is the numerical aperture of the imaging optics. This approximation also assumes that $S_{F R E T}$ is large enough to include many hairpins.

The reason why this resolution limit is not affected by the UV wavelength is because the UV beam is transiently turned on only when a hairpin folds in a target spot whose location precision is not related to the UV beam in any way. The number of DNA hairpins within a FRET excitation beam would be $d_{D N A} S_{F R E T}$, where $d_{D N A}$ is the surface density of DNA in $\mathrm{m}^{-2}$. Subsequently, the minimum time window during which each hairpin folds once should be approximately $\tau_{\text {folded }} d_{D N A} S_{F R E T}$. This time should be the minimum photobleaching lifetime required for each hairpin folds once in the FRET beam.

$\tau_{\text {photobleaching }} \approx d_{D N A} \tau_{\text {folded }} S_{\text {FRET }}$ 
With a fixed photobleaching lifetime, the maximum DNA density on the surface

$d_{D N A} \approx \frac{\tau_{\text {photobleaching }}}{\tau_{\text {folded }} S_{\text {FRET }}}$

As the resolution limit would be the average distance between DNA hairpins,

$$
R=\sqrt{\frac{1}{d_{D N A}}} \approx \sqrt{\frac{\tau_{\text {folded }} S_{F R E T}}{\tau_{\text {photobleaching }}}}
$$

Finally, the signal integration time (=camera exposure time) should be half of the average folded state lifetime so that a folded hairpin can be UV cured in the next exposure.

Therefore,

$$
R \approx \sqrt{\frac{\tau_{\text {folded }} S_{F R E T}}{\tau_{\text {photobleaching }}}}=\sqrt{\frac{\left(\frac{E}{2}\right) S_{F R E T}}{\tau_{\text {photobleaching }}}}=\sqrt{\frac{\left(\frac{E}{2}\right)\left(\pi\left(\frac{\lambda_{F R E T}}{2 N . A .}\right)^{2}\right)}{\tau_{\text {photobleaching }}}}=\frac{1}{2} \sqrt{\frac{\pi E}{2 \tau_{\text {photobleaching }}}} \frac{\lambda_{F R E T}}{N . A .}
$$

The camera exposure time $E$ in our current setup is $100 \mathrm{~ms}$ to achieve a fluorophore localization precision higher than the SOLiD resolution limited by the other factors and to ensure near complete crosslinking. The current theoretical limit with $100 \mathrm{~ms}$ exposure at $23 \mathrm{kHz}$ photon detection rate is $5.4 \mathrm{~nm}$ with $N . A .=1.4$. This photon detection rate is set to result in the localization precision of $5.4 \mathrm{~nm}$ per $100 \mathrm{~ms}$ according to a formula $\frac{\lambda}{2 N \cdot A \cdot} \cdot \frac{1}{\sqrt{N}}{ }^{4}$, where $\lambda$ is the FRET acceptor detection wavelength $(\approx 720 \mathrm{~nm})$ and $N$ is the number of photons detected $(=2300)$, thereby not limiting the SOLiD resolution. A total of $3 \times 10^{7}$ photons emitted before photobleaching under our typical conditions and $15 \%$ detection efficiency at $720 \mathrm{~nm}$ result in the photobleaching lifetime of $196 \mathrm{sec}$. 
Figure S1. Characterizations of CNV-NHS-ester. (A) ${ }^{1} \mathrm{H}$ NMR, (B) ${ }^{13} \mathrm{C}$ NMR, and (C) LC-MS analysis. Chemical shifts in NMR spectra are reported as $\delta$-values in ppm.

Coupling constants are provided in $\mathrm{Hz}$. All given ${ }^{13} \mathrm{C}$ spectra are proton-decoupled. LCMS analysis indicates two major components consisting of the CNV-NHS-ester (RT: $2.54 \mathrm{~min},[\mathrm{M}+\mathrm{H}]$ found 374.1083 ) and the corresponding carboxylate (RT: $2.13 \mathrm{~min}$, $[\mathrm{M}+\mathrm{H}]$ found 277.0933). With no indication of the presence of the carboxylate in either NMR spectra, the appearance of this peak must be the result of acid-catalyzed hydrolysis of the labile NHS ester under the given elution conditions. LC-MS analysis indicates $>90 \%$ purity of the starting material as quantified by relative peak area. Elution parameters: Solvent A; $100 \% \mathrm{dH}_{2} \mathrm{O}, 0.1 \%$ formic acid. Solvent B; $100 \%$ acetonitrile, $0.1 \%$ formic acid. $0-2 \mathrm{~min} 40 \% \mathrm{~B}(0.3 \mathrm{~mL} / \mathrm{min}) ; 2-5$ min gradient $40 \%$ to $90 \% \mathrm{~B}(0.3$ $\mathrm{mL} / \mathrm{min}) ; 5-8.5 \mathrm{~min} 90 \% \mathrm{~B}(0.3 \mathrm{~mL} / \mathrm{min}), 8.5-9.5 \mathrm{~min}$ gradient $90 \%$ to $40 \% \mathrm{~B}(0.3$ $\mathrm{mL} / \mathrm{min}) ; 9.5-10 \mathrm{~min} 40 \%$ B $(0.3 \mathrm{~mL} / \mathrm{min})$.

\section{A}

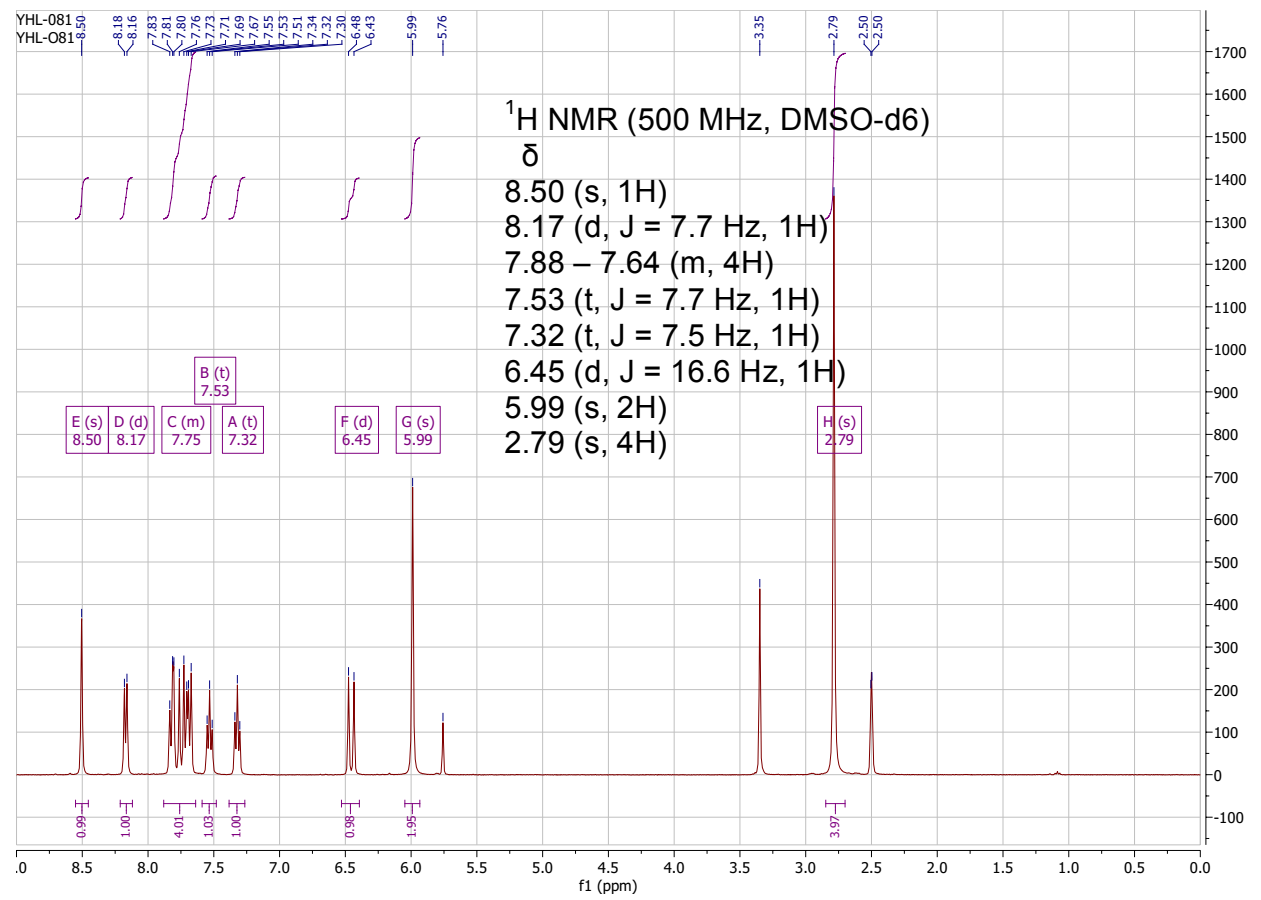


B

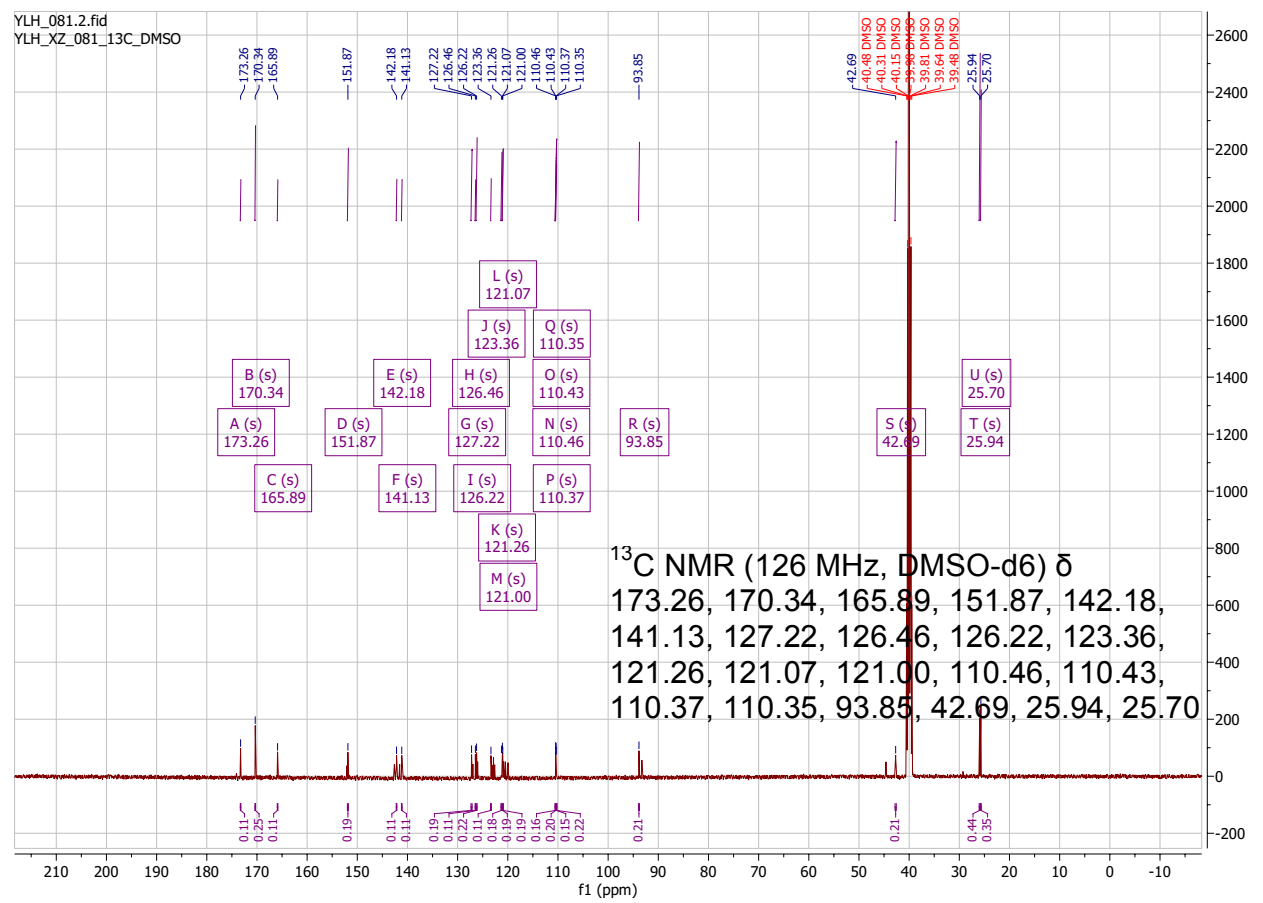

C

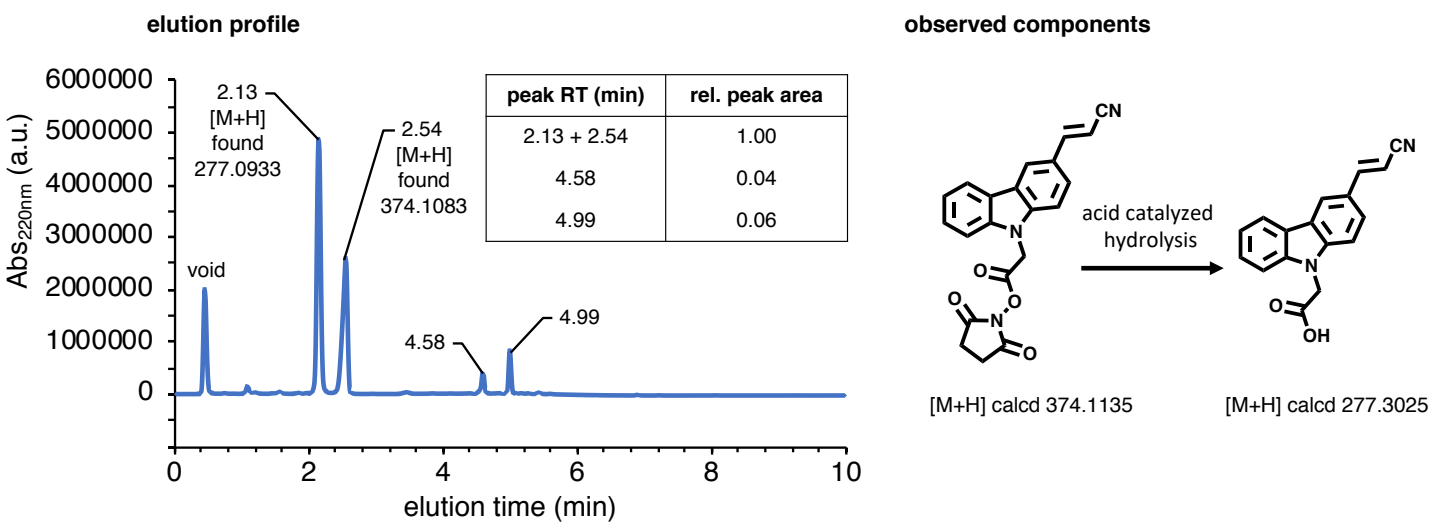

HRMS: RT 2.47-2.56 min, ESI (pos)

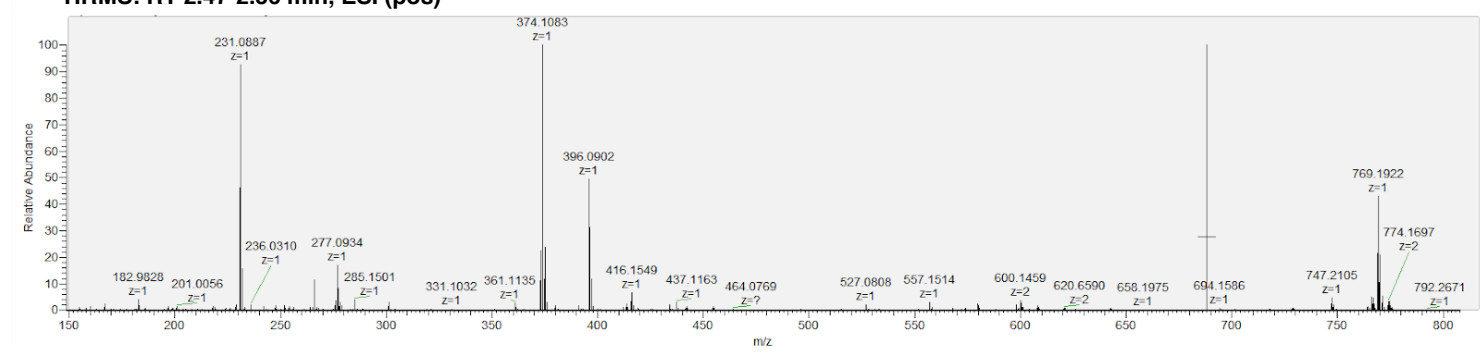


Figure S2. The sequences of the anchor, pin, bond, and effector DNA strands (Fig. 1A), and strands 1/2/3/4 (Fig. 3B). The fluorophores (Cy3 and Cy5) and an amine-modifier (Uni-Link $^{\mathrm{TM}}$ amino modifier, UniAmM) were inserted during synthesis (Integrated DNA Technologies, Inc. Coralville IA).

SOLiD (Fig. 1A)

Anchor strand GGCTGGTGTGGCCTGAATAATCGCTGCACGAGTT/iCy3/GACCGAGGAAGGTTTT/3Bio/

Pin strand

/5Cy5/GCA/iUniAmM/GCGTAAATAAAATAAAAATAAAAAATAATAAAAAAACAACTT CCGTTCGGACCCGGC

Bond strand

CAGGCCACACCAGCCAAAAGCCGGGTCCGAACGG

Effector strand

/5Biosg/GCCGGGTCCGAACGGAAGTTG

Crosslinker performance test (Fig. 3B)

Strand 1

GATTCGATTGCTCCTGGCTAGTTACCGCACCCGTGTTTT/3Bio/

Strand 2

CACGGGTGCGGTAACTAGCCAGGAGCAATCGAATCTTG ACGGTGCA GT

Strand 3

GGTCCTCCAGGTTGTGGTTACTGCA/iUniAmM/CCGTCAA

Strand 4

CCACAACCTGGAGGACCTTTTTTTTTTTT/3Bio/ 
Figure S3. PAGE analyses show complete DNA hybridization. The DNA fragments shown in figures 1A and 3B (see figure S2 for their sequences) were hybridized in the stoichiometric quantities and analyzed on a native PAGE gel under standard conditions, showing complete hybridization in both cases. (A) DNA hybridization of the hairpin construct shown in figure 1A. (B) Hybridization of the construct shown in figure 3B. Note that the ladder DNA components are double-stranded and shown only for relative comparison among the fragments.
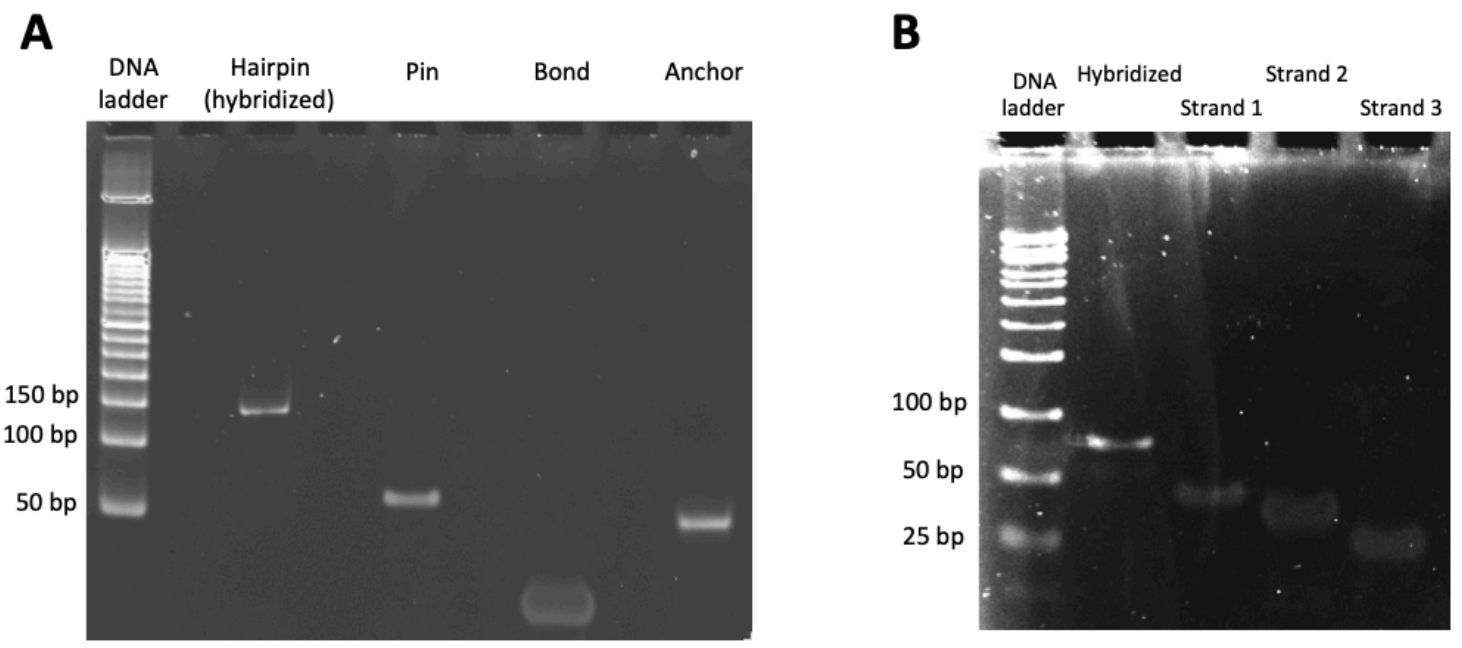
Figure S4. SOLiD patterning in the absence of the oxygen scavenger system Protocatechuate dioxygenase (PCD) and Protocatechuic acid (PCA). The experimental conditions were kept the same as those in figure 4 except for that $\mathrm{PCD} / \mathrm{PCA}$ is absent here. (A) The FRET donor fluorescence intensity averaged from 50 donors selected at 5 different locations on the slide surface is plotted against FRET excitation duration. The photobleaching lifetime is $3.9 \mathrm{sec}$. Accordingly, the FRET excitation duration to photobleach $>99 \%$ of the fluorophores is $18 \mathrm{sec}$. (B) SOLiD performs poorly with 3.9 sec photobleaching lifetime. For patterning, FRET excitation was kept on for $20 \mathrm{sec}$ at each spot shown in figure $4 \mathrm{C}$ in order to ensure $>99 \%$ photobleaching before moving on to the next spot. The image displays the area where the SOLiD patterning was performed, showing no sign of the "U" pattern. This result verifies that the photobleaching lifetime is a crucial determinant of the SOLiD performance and resolution.

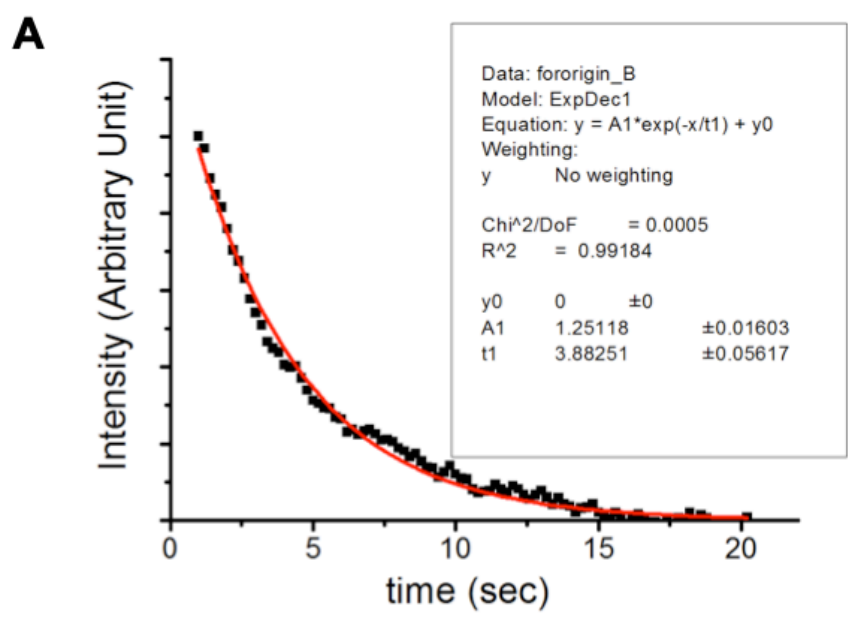

B

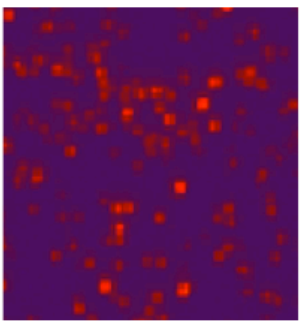




\section{References}

1. Yoshimura, Y.; Fujimoto, K., Ultrafast Reversible Photo-Cross-Linking Reaction: Toward in Situ DNA Manipulation. Org Lett 2008, 10 (15), 3227-3230.

2. Sakamoto, T.; Tanaka, Y.; Fujimoto, K., DNA Photo-Cross-Linking Using 3Cyanovinylcarbazole Modified Oligonucleotide with Threoninol Linker. Org Lett 2015, 17 (4), 936-939.

3. Lee, J.; Crickard, J. B.; Reese, J. C.; Lee, T. H., Single-molecule FRET method to investigate the dynamics of transcription elongation through the nucleosome by RNA polymerase II. Methods (San Diego, Calif.) 2019.

4. von Diezmann, A.; Shechtman, Y.; Moerner, W. E., Three-Dimensional Localization of Single Molecules for Super-Resolution Imaging and SingleParticle Tracking. Chem Rev 2017, 117 (11), 7244-7275. 